\title{
Real space-time imaging of valence electron motion in molecules
}

Manish Garg ( $\square$ mgarg@fkf.mpg.de )

Max Planck Institute for Solid State Research

Alberto Martin-Jimenez

Max Planck Institute for Solid State Research

Michele Pisarra

Instituto Madrileño de Estudios Avanzados en Nanociencia (IMDEA Nano)

Fernando Martín

Universidad Autonoma de Madrid https://orcid.org/0000-0002-7529-925X

Klaus Kern

Max Planck Institute for Solid State Research

\section{Physical Sciences - Article}

Keywords: valence electron motion, scanning tunnelling microscopy, attosecond technologies

Posted Date: November 5th, 2020

DOI: https://doi.org/10.21203/rs.3.rs-99925/v1

License: (c) (1) This work is licensed under a Creative Commons Attribution 4.0 International License.

Read Full License 


\title{
Real space-time imaging of valence electron motion in molecules
}

\author{
M. Garg ${ }^{1 *+}$, A. Martin-Jimenez ${ }^{1+}$, M. Pisarra ${ }^{2}$, F. Martín ${ }^{2,3,4}$, K. Kern ${ }^{1,5}$ \\ ${ }^{1}$ Max Planck Institute for Solid State Research, Heisenbergstr. 1, 70569 Stuttgart, Germany \\ 2 Instituto Madrileño de Estudios Avanzados en Nanociencia (IMDEA Nano), Faraday 9, \\ Cantoblanco, 28049 Madrid, Spain \\ ${ }^{3}$ Departamento de Química, Módulo 13, Universidad Autónoma de Madrid, 28049 Madrid, Spain \\ ${ }^{4}$ Condensed Matter Physics Center (IFIMAC), Universidad Autónoma de Madrid, 28049 Madrid, \\ Spain \\ ${ }^{5}$ Institut de Physique, Ecole Polytechnique Fédérale de Lausanne, 1015 Lausanne, Switzerland
}

*Author to whom correspondence should be addressed.

${ }^{+}$Authors with equal contribution.

Tracking valence electron motion in molecules is the key to understand and control chemical transformations ${ }^{1-4}$. Contemporary techniques in attosecond science $^{5-8}$ have the capability to generate and track the consequences of this motion in real time, but not in real space. Consequently, the local time-evolution of the electron density can so far only be inferred by reconstruction from the (often elusive) features appearing in electron, ion, absorption or emission spectra ${ }^{9-12}$, mainly in small molecules. Scanning tunnelling microscopy $^{13}$ (STM), on the other hand, can locally probe the valence electron density in molecules, but cannot provide by itself dynamical information at this ultrafast time scale. Here, we demonstrate that, by combining STM and attosecond technologies, the dynamics of a coherent superposition of valence electronic states generated by $<6$ femtosecond long carrier-envelope-phase (CEP) stable laser pulses can be directly and simultaneously visualized with picometer spatial resolution and 300 attosecond temporal resolution, thus going well beyond the previously established space-time limits ${ }^{14}$ and bypassing any kind of reconstruction. In particular, we show that near fields of near-infrared pulses confined to the apex of a nanotip of an STM enable concurrent real-space and real-time imaging of the valence electron dynamics generated in the perylenetetracarboxylic dianhydride (PTCDA) molecule, as well as exerting full control of the electron density in the highest occupied (HOMO) and lowest unoccupied (LUMO) molecular orbitals. We envision that this approach could thus open the way to the unambiguous observation and manipulation of electron dynamics in complex molecular systems, in monolayer thick $2 \mathrm{D}$ materials ${ }^{15}$ and in superconductors ${ }^{16}$.

Light-matter interactions at the atomic and molecular scales offer the possibility to probe electronic motion at its nature length ${ }^{17-20}$ (sub- $\AA$ ) and time scales ${ }^{21}$ (100 as to few fs). Lightwave-driven STM can now generate and track extremely fast tunnelling currents ${ }^{21-28}$, as well as coherently control electron tunnelling on $\sim 200$ as timescale ${ }^{21}$ and switching in molecules ${ }^{29}$. Yet, the ultimate goal, to watch electrons in action in real-space and real-time, still 
has remained elusive ${ }^{5,30}$. Here we combine CEP stable few-cycle $(<6 \mathrm{fs}$ long) laser pulses with an STM to break this fundamental barrier, and to follow electron motion concurrently with pm spatial and 300 as temporal resolutions.

In our experiments, orthogonally linearly polarised near infrared laser pulses with slightly different carrier frequencies, obtained by selecting the $0^{\text {th }}$ and $1^{\text {st }}$ order diffraction beams of the laser pulses passing through an acousto-optic-frequency-shifter (AOFS), were focused at the apex of a W nanotip in tunnel contact with molecular layers of Perylenetetracarboxylic dianhydride (PTCDA) grown on top of a Au (111) surface. The polarization axes of the two laser pulses formed an angle of $45^{\circ}$ with respect to the tip axis and their incidence angle at the junction was $7^{\circ}$ with respect to the Au (111) surface (see Fig. 1a and section I in methods for details of the experimental set-up). This configuration ensures that the non collinear polarizations of the pulses only overlap with each other through their component along the nanotip axis; this overlap occurs within the small bandwidth of the STM. In this way, we circumvent the use of mechanical modulation to lock-in detect the laser-induced tunneling current in the STM and avoid spatial interferences between the laser pulses, which can make the tunnel junction unstable ${ }^{31}$. The correlation of the laser-induced tunneling current as a function of the delay between the two laser pulses is shown in Fig. 1d. The orange curve shows the variation in the tunnel gap width during the measurement. At zero delay between the two laser pulses, the polarization induced along the nanotip axis resembles the one induced by a single laser pulse at the tunnel junction at the small offset frequency (see section II in methods). This induced polarization along the tip axis can be assumed to be instantaneous ${ }^{32}$ for the timescale of the dynamics presented here. The excellent stability of our interferometric pumpprobe set-up over several hours (Fig. S2, methods) enables the possibility to lock-in detect the tunneling current dynamics induced by a single laser pulse, without the need to mechanically modulate the laser pulses. It is important to note that, for the wavelength $(\lambda \sim 850 \mathrm{~nm})$ and intensities $\left(\sim 1 \times 10^{12} \mathrm{~W} / \mathrm{cm}^{2}\right)$ of the laser pulses used in this work, only photon-driven tunneling is possible ${ }^{21}$ (Keldysh parameter, $\gamma>1$ ), not field-driven tunneling as it would be the case at higher pulse intensities ${ }^{21}$.

The electronic energy levels of a single monolayer of PTCDA molecules are favorably positioned with respect to the surface state of $\mathrm{Au}$ (111). A single photon of $\sim 1.5 \mathrm{eV}$ energy from 
the laser pulse resonantly couples the highest occupied molecular orbital (HOMO) to the $\mathrm{Au}$ (111) surface state as well as the latter to the lowest unoccupied molecular orbital (LUMO) of PTCDA. Thus, with controlled positioning of the electronic levels of the molecule with respect to the Fermi level of the nanotip, enabled by adjustment of the bias at the tunnel junction, a laser pulse can either induce a transition from the HOMO to the surface state, Fig. 1b, or from the surface state to the LUMO, Fig. 1c. In other words, by modulating the bias voltage at the tunnel junction one can control the initial state from which the laser-induced transition takes place.

We consider first the case of zero delay between the two laser pulses (hereafter referred to as a single-pulse experiment). We note that, in this case, the electric-field components of the two pulses cancel each other in the surface plane due to the specific geometric arrangement of the two laser pulses (Fig. 1a), so that the only active electric-field component is that along the tip axis (this is no longer true for non-zero delay between the pulses). As soon as this single pulse reaches the sample, a transfer of population from the lower to the upper level starts. Fig. 1e shows the calculated temporal evolution of the populations in the surface and LUMO states resonantly coupled by our 6 fs long laser pulse obtained from standard two-level Rabi formulas (see section $\mathrm{V}$ in methods). As can be seen, at the end of the laser pulse, most of the electronic population remains in the lower state, thus excluding the possibility that a single 6 fs pulse induces Rabi oscillations between those states. This is the obvious consequence of the fact that, for the peak electric field $E$ of the laser pulse used in the experiment and the small value of the dipole coupling between the two levels, $\mu$ (see section VI in methods), the Rabi period, $2 \pi \hbar /(\mu E)$, is much longer than the pulse duration. We note that the transition dipole moment along the tip axis (the only active component of $\mu$ in the single-pulse case) is small due to the nearly perfect planar arrangement of the Au (111) atoms and the PTCDA layer. We exclude any further exchange of population between the two states when the pulse is over due to the extremely short tunneling times ${ }^{21}$ ( $<0.5 \mathrm{fs}$ ), from the sample to the STM junction. Therefore, in a single-pulse experiment, tunneling from the sample to the $\mathrm{W}$ tip will essentially carry spatial information about the lower electronic state. Spatially resolved topographic scans of the single-pulse induced tunneling current at various bias settings at the tunnel junction are shown in Fig. 1f to Fig. 1i. 
As can be seen, when the HOMO of the PTCDA molecules is aligned with the Fermi level of the W nanotip, the laser-driven tunneling current images the spatial profile of the HOMO orbitals (Fig. 1f). When it is the Au (111) surface state that is aligned with the Fermi level, the majority of the laser-driven tunneling current arises from the surface state, so that the intensity of the current is much higher on the metallic surface than on the molecules (Fig. 1g). At a bias where not one, but two dipole transitions are possible, e.g., one transition from the surface state to the LUMO and another from the Fermi level of the tip to the LUMO (processes (1) and (2) in Fig. 1c), the spatial profile of the laser-induced tunneling current exhibits large intensities on both the molecule and the Au (111) surface, Fig. 1h (see also Fig. S3 in methods). At a bias voltage where the Fermi level of the W nanotip is lifted above the LUMO of the molecule, the dominant process should be a transition from the Fermi level of the tip to an empty state of the molecules below the tunnel barrier. In this case, the spatial distribution of the current should not carry, in principle, any information on the electronic levels of the molecule. However, at a bias-voltage of $1.4 \mathrm{~V}$, the spatial distribution of the laser-induced tunneling current resembles the distribution of the LUMO of the PTCDA molecules as shown in Fig. 1i (see also section III in methods). This is due to the elastic tunneling of electrons from the nanotip to the molecule [or to the Au (111) surface state] prior to the interaction with the laser pulse. Based on the above arguments, the laser-driven imaging of the surface state and the LUMO has to be symmetric around a range of bias voltages at the tunnel junction, from $-200 \mathrm{mV}$ to $+200 \mathrm{mV}$, due to the fact that we access the same initial and final states (see Fig. S3 and S4 in methods). Fig. S3 and Fig. S4 in methods compare the spatial topography obtained by the constant tunneling current operation mode in the STM with the simultaneously acquired spatial distribution of the laser-induced tunneling current. The acquired surface topographies have absolutely no variation in their profile except for the voltages where we align the resonance of the electronic levels with the Fermi level of the tip or the sample, in sharp contrast to the spatial distribution profile of the laser-induced tunneling current, connoting strongly to the dipole-coupled interaction picture between the electronic levels presented above. Comparison of simulated STM images utilizing the Tersoff-Hamann ${ }^{33}$ approach and DFT calculations (see section VI in methods) with the experimentally measured images for a bias voltage of $-1.8 \mathrm{~V}$ (HOMO), $+1.4 \mathrm{~V}$ (LUMO) and $-200 \mathrm{mV}$ is shown in Fig. S5. At a bias voltage of $-200 \mathrm{mV}$, a tunneling current between the molecules becomes apparent (Fig. S5d), which arises 
due to the presence of the surface state of $\mathrm{Au}$ (111) at this bias, in agreement with the experiments (Fig. 1h and Fig. S5c).

In order to visualize electron dynamics involving two dipole-coupled states of the PTCDA/Au (111) system, we have varied the delay between the two orthogonally polarized laser pulses (hereafter called pump and probe pulses) and performed space-resolved topographic scans of the laser-induced tunneling current. For the laser parameters used in this work and the specific electronic states involved in the process, the optimum conditions to induce such dynamics correspond to the case in which the pump and probe pulses overlap in time. In this case, the populations for the upper $(u)$ and lower $(l)$ states as a function of the pump-probe delay $\tau$ are approximately given by (see section $\mathrm{V}$ in methods):

$$
\begin{aligned}
& \wp_{l}(T)=\cos ^{2}\left[\frac{\mathcal{E} T}{\hbar}\left\{\left|\mu_{l u}^{\|}\right|^{2} \sin ^{2}\left(\frac{\omega_{u l} \tau}{2}\right)+\left|\mu_{l u}^{\perp}\right|^{2} \cos ^{2}\left(\frac{\omega_{u l} \tau}{2}\right)\right\}^{1 / 2}\right] e^{-\Gamma \tau / \hbar} \\
& \wp_{u}(T)=\sin ^{2}\left[\frac{\mathcal{E} T}{\hbar}\left\{\left|\mu_{l u}^{\|}\right|^{2} \sin ^{2}\left(\frac{\omega_{u l} \tau}{2}\right)+\left|\mu_{l u}^{\perp}\right|^{2} \cos ^{2}\left(\frac{\omega_{u l} \tau}{2}\right)\right\}^{1 / 2}\right] e^{-\Gamma \tau / \hbar}
\end{aligned}
$$

where $\mathcal{E}=E / \sqrt{2}$, with $E$ being the peak electric field of the laser pulse, $T$ is the pulse duration, $\hbar \omega_{u l}$ is the energy difference between the $u$ and $l$ states, $\Gamma$ is their energy width, and $\hbar$ is Planck's constant. $\mu_{l u}^{\|}$and $\mu_{l u}^{\perp}$ are the transition dipole-moment matrix elements between the $u$ and $l$ states along the parallel $(\|)$ and perpendicular $(\perp)$ directions with respect to the molecular plane (parallel to the Au (111) surface). For the electronic levels of the PTCDA molecules on top of the Au (111) surface, $\Gamma=0.4 \mathrm{eV}$, which is due to dielectric screening of the PTCDA electrons by the sea of metal electrons. The effect of $\Gamma$ is to induce a decay of the electronic coherence induced by the laser pulses. We note that for zero delay ( $\tau=0$, single-pulse case) and no width $(\Gamma=0)$, these formulas are identical to the well-known Rabi equations for a two-level system ${ }^{34}$.

The arrangement of the electronic energy levels of one monolayer of PTCDA molecules on top of $\mathrm{Au}$ (111) as measured by scanning tunneling spectroscopy (STS) is shown in Fig. 2a. According to equation (1), by properly lifting the HOMO of the PTCDA molecules above the Fermi level of the nanotip (Bias $=-1.8 \mathrm{~V}$ ), an impinging photon from the pump pulse will coherently couple the HOMO and the surface state. A probe pulse at a certain delay from the pump pulse will then induce a transition between the two states. If the electronic density is concentrated in the surface state, it will stimulate the transition back to the HOMO and vice-versa, akin to quantum beating between 
the two states (see section V in methods for more details). In this way the electronic density will resemble that of either one of the two states depending on the delay between the two pulses, and electrons will eventually tunnel carrying the spatial information of the state where they tunnel from. A schematic illustration of this process is shown in Fig. 2b. At zero delay $\left(\tau_{1}\right)$ between the two pulses, the spatially resolved laser-induced imaging maps the spatial profile of the HOMO (Fig. 2d), whereas at a delay of 1.2 fs $\left(\tau_{2}\right)$ the intensity of the tunnel current maps the Au (111) surface state in between the molecules (Fig. 2c). The laser-induced tunneling current for the latter is much higher than for the former, which is consistent with the single-pulse spatial imaging discussed above (Fig. 1g).

The spatially resolved laser-induced tunneling current as a function of the delay between pump and probe pulses is shown in Fig. 2e. A clear oscillation of the electron density between the HOMO of the PTCDA molecules and the Au (111) surface state can be seen in the space-time snapshots, with an oscillation period of $\sim 2.7 \mathrm{fs}$. Details of the experimental methodology used to record these space-time maps are given in section IV of the methods. Briefly, in the pump-probe measurement, when the population between the two states oscillates out of phase as a function of delay, the topographic scan of the laser-induced tunneling current at a fixed delay will have information primarily from either of the two states. By performing, an amplitude-selective filtering of the spatial distribution of the laser-induced tunneling current in each space-time scan one can transparently distinguish the electronic population in either of the states as a function of the delay, owing to the sharp contrast of the spatial distribution of the laser-induced tunneling current arising from the two states. Integration of the laser-induced tunneling current from the individual panels in Fig. 2e reveals the population dynamics of electrons between the two states as shown in Fig. $2 \mathrm{f}$. The dynamics predicted by the two-level model given by equation (1), using the present laser parameters, an electronic decoherence time of $\sim 5 \mathrm{fs}$ and a transition dipole-moment matrix elements of 1 a.u. $\left(\mu_{l u}^{\|}\right)$and 0.2 a.u. $\left(\mu_{l u}^{\perp}\right)$ along the parallel and perpendicular axis of the PTCDA molecules, respectively, compatible with the values obtained from the density functional theory (DFT) simulations reported in section VI of the methods, are in excellent agreement with the experimental findings (see dotted lines in Fig. 2f).

Electron dynamics arising from the interaction of the laser pulses with a monolayer of PTCDA molecules on $\mathrm{Au}$ (111) involves both molecular and surface states. In order to observe pure 
molecular electron dynamics, we have grown multilayers of PTCDA molecules on top of the Au (111) surface. Weak van der Waals coupling between the molecular layers ensures that the upper layer can be sufficiently decoupled from the $\mathrm{Au}$ (111) surface state, so that the latter can neither contribute to the induced dynamics nor to the tunneling current ${ }^{35}$. We have found that these conditions are met for four or more PTCDA monolayers. The scanning tunneling spectrum for the 4-monolayer system is shown in Fig. 4a. As can be seen, there is no longer any trace of the surface state and the HOMO-LUMO gap is of the order 2.5-3.0 eV. This energy gap can be overcome by single-photon transitions involving the blue tail of the pulses or two-photon transitions ${ }^{21,36}$ involving the pulses' peak frequency. However, in the present weak-field (perturbative) conditions and in the absence of the surface state, which precludes resonant enhanced multiphoton processes, two-photon transitions should be much less likely than one-photon transitions. Hence, according to equation (1), when a laser pulse interacts with upper-layer PTCDA molecules whose HOMOs have been lifted close to the Fermi level of the nanotip (-1.8 V), oscillations between HOMO and LUMO, i.e. between pure molecular states, are expected, but with a frequency that will be significantly different from that of the one-monolayer case due to a different energy gap. This strategy allows for laser-induced spatial imaging of the frontier molecular orbitals as shown in Fig. 3e and Fig. 3f. At variance with the one-monolayer case, not all molecules lead to the same laserinduced tunneling current: half of the molecules, organized in parallel rows, lead to considerably higher currents than the other half. The rows reflecting the HOMOs are accompanied by rows with very low laser-induced tunneling current in the LUMOs (Fig. 3f). In contrast, when the LUMOs of the latter rows become apparent, the HOMOs in the former rows are nearly invisible (Fig. 3e). As shown by extensive DFT calculations for the four-monolayer PTCDA/Au (111) system (see section VI in methods), this behavior is the consequence of the specific vertical stacking of the PTCDA molecules: the delicate balance between intra-layer hydrogen bonding and inter-layer $\pi$ $\pi$ interactions forces PTCDA molecules in the higher layers to displace horizontally $(\sim 1 \AA)$ with respect to molecules in the lower layers. This leads to a local density of states (DOS) where molecules in one of the rows have a higher DOS than those in the adjacent rows (see Fig. 4a and section VI in methods). Figs. 3a and 3b show simulated STM images resulting from the TersoffHamann approach ${ }^{33}$ applied to the fully relaxed four-monolayer PTCDA/Au (111) system described by DFT (see section VI in methods). As the calculated images are obtained with infinite spatial resolution, comparison with experiment requires convolution with the wavefunction of the 
nanotip, which can be modelled by an s-wave with decay lengths of 1.8 and $0.8 \AA$ along the vertical and horizontal directions, respectively. The results shown in Figs. 3c and $3 \mathrm{~d}$ are in excellent agreement with the experimentally measured spatial profiles. It is worth noting that infrared laser pulses can achieve pm spatial resolution on their integration with an STM, several orders of magnitude below their diffraction limit ${ }^{37,38}$. The spatial distribution of the laser-induced tunneling current in the case of the HOMO is within the molecule, whereas in the case of the LUMO, the distribution is primarily located at the edges of the molecule. The HOMO has an oval shape compared to the rectangular shape of the LUMO.

Real space-time images as a function of the delay between the pump and probe pulses are shown in Fig. 4d. Pronounced oscillation of the electron density between the HOMO and the LUMO levels can be observed. The temporal evolution of the populations in HOMO and LUMO levels as a function of the pump-probe delay, obtained by integrating the individual panels in Fig. 4d, are shown in Fig. 4e. Counter-phase oscillations of the HOMO and LUMO populations with a period of $\sim 1.4$ fs can be clearly seen. This is approximately half the period of the oscillation observed in the one-monolayer case (Fig. 2f) and corresponds to a frequency that matches the energy separation between the HOMOs and the LUMOs in the four-monolayer system. Both the counterphase character of the oscillations and their frequency are very well reproduced by the two-level formula ( equation 1) in which the experimental HOMO-LUMO gap, an electronic decoherence time of $\sim 10 \mathrm{fs}$ and a value of the transition dipole moment of 0.8 a.u. $\left(\mu_{l u}^{\|}\right)$and 0.1 a.u. $\left(\mu_{l u}^{\perp}\right)$ were used. The electronic decoherence time in this case is significantly longer than for the monolayer of PTCDA on Au (111), which primarily arises from the reduced dielectric screening from the metal surface. The values of the transition dipole moment are compatible with those obtained from our DFT calculations (see section VI in methods). In this case, the only components of the transition dipole moments that are significantly different from zero lie in the molecular plane (for perfectly planar PTCDA layers in which the molecules in different layers lie on top of each other, the transition dipole moment in the direction perpendicular to the plane would be exactly zero due to symmetry selection rules). Thus, dipole couplings between molecular states are possible because the polarization axis of the laser pulses is at $45^{\circ}$ with respect to the molecular plane (Fig. 1a). At variance with the one-monolayer case, the amplitude of the measured oscillations does not decrease monotonously with the pump-probe delay. This is most likely due to the competition between one- and two-photon transitions, as well as to the fact that the HOMO and LUMO bands 
are not as flat as in the one-monolayer case, thus allowing for the effective involvement of more than two electronic levels (multiple HOMOs and LUMOs) in the induced dynamics.

The capability demonstrated in this work to image electron dynamics simultaneously at its natural length and natural time scales opens completely new avenues to understand chemical transformations driven by electron transfer in, e.g., photosynthetic molecules and other light harvesting molecules ${ }^{39,40}$, and to the unambiguous observation of electron dynamics in complex molecular systems, 2D materials and superconductors. Furthermore, the high degree of control that can be exerted in the population of the molecules' frontier orbitals by just varying the delay between identical, orthogonally polarized, temporally overlapping pulses suggests that a similar approach might be useful to drive chemical reactions occurring on surfaces, which can bring new scenarios for heterogeneous catalysis and for ultrafast dynamics of charge transfer in molecules ${ }^{41}$. Direct imaging (at the orbital scale) of a transition state of a molecule undergoing a chemical transformation is also realizable in near future.

\section{Contributions}

M. G. and K.K. conceived the project and designed the experiments, K.K. supervised the project. M.G. and A.M.J built the experimental set-up, performed the experiments and analyzed the experimental data. M.P. and F.M. developed the analytical two-state model and performed the DFT calculations. M.P., M.G. and F.M. analyzed the theoretical data. All authors interpreted the results and contributed to the preparation of the manuscript.

\section{Acknowledgments}

We thank Rico Gutzler for fruitful discussions, and Wolfgang Stiepany for technical support. Work supported by the European COST Action AttoChem. All calculations were performed at the Mare Nostrum Supercomputer of the Red Española de Supercomputación (BSC-RES) and the Centro de Computación Científica de la Universidad Autónoma de Madrid (CCC-UAM). MP and FM acknowledge support by the MICINN projects PID2019-105458RB-I00, the "Severo Ochoa" Programme for Centres of Excellence in R\&D (SEV-2016-0686), the "María de Maeztu" Programme for Units of Excellence in R\&D (CEX2018-000805-M) and the Comunidad de Madrid Synergy Grant FULMATEN. 


\section{References}

1 Hockett, P., Bisgaard, C. Z., Clarkin, O. J. \& Stolow, A. Time-resolved imaging of purely valence-electron dynamics during a chemical reaction. Nat. Phys. 7, 612-615 (2011).

2 Worner, H. J., Bertrand, J. B., Kartashov, D. V., Corkum, P. B. \& Villeneuve, D. M. Following a chemical reaction using high-harmonic interferometry. Nature 466, 604-607 (2010).

3 Gessner, O. et al. Femtosecond multidimensional imaging of a molecular dissociation. Science 311, 219-222 (2006).

4 Li, W. et al. Time-Resolved Dynamics in N2O4 Probed Using High Harmonic Generation. Science 322, 1207-1211 (2008).

5 Corkum, P. B. \& Krausz, F. Attosecond science. Nat. Phys. 3, 381-387 (2007).

6 Krausz, F. \& Ivanov, M. Attosecond physics. Rev. Mod. Phys. 81, 163-234 (2009).

7 Sansone, G. et al. Electron localization following attosecond molecular photoionization. Nature 465, 763-U763 (2010).

8 Itatani, J. et al. Tomographic imaging of molecular orbitals. Nature 432, 867-871 (2004).

9 Goulielmakis, E. et al. Real-time observation of valence electron motion. Nature 466, 739743 (2010).

10 Calegari, F. et al. Ultrafast electron dynamics in phenylalanine initiated by attosecond pulses. Science 346, 336-339 (2014).

11 Jordan, I. et al. Attosecond spectroscopy of liquid water. Science 369, 974- (2020).

12 Nisoli, M., Decleva, P., Calegari, F., Palacios, A. \& Martin, F. Attosecond Electron Dynamics in Molecules. Chem Rev 117, 10760-10825 (2017).

13 Binning, G., Rohrer, H., Gerber, C. \& Weibel, E. Surface Studies by Scanning Tunneling Microscopy. Phys. Rev. Lett. 49, 57-61 (1982).

14 Li, Z., Gyawali, S., Ischenko, A. A., Hayes, S. \& Miller, R. J. D. Mapping Atomic Motions with Electrons: Toward the Quantum Limit to Imaging Chemistry. Acs Photonics 7, 296320 (2020).

15 Poellmann, C. et al. Resonant internal quantum transitions and femtosecond radiative decay of excitons in monolayer WSe2. Nat Mater 14, 889-+ (2015).

16 Mitrano, M. et al. Possible light-induced superconductivity in K3C60 at high temperature. Nature 530, 461 (2016). 
17 Lee, J., Crampton, K. T., Tallarida, N. \& Apkarian, V. A. Visualizing vibrational normal modes of a single molecule with atomically confined light. Nature 568, 78-82 (2019).

18 Jaculbia, R. B. et al. Single-molecule resonance Raman effect in a plasmonic nanocavity. Nat Nanotechnol 15, 105 (2020).

19 Yang, B. et al. Sub-nanometre resolution in single-molecule photoluminescence imaging. Nat. Photonics (2020).

20 Doppagne, B. et al. Electrofluorochromism at the single-molecule level. Science 361, 251254 (2018).

21 Garg, M. \& Kern, K. Attosecond coherent manipulation of electrons in tunneling microscopy. Science 367, 411 (2020).

22 Cocker, T. L. et al. An ultrafast terahertz scanning tunnelling microscope. Nat. Photonics 7, 620-625 (2013).

23 Cocker, T. L., Peller, D., Yu, P., Repp, J. \& Huber, R. Tracking the ultrafast motion of a single molecule by femtosecond orbital imaging. Nature 539, 263-267 (2016).

24 Jelic, V. et al. Ultrafast terahertz control of extreme tunnel currents through single atoms on a silicon surface. Nat. Phys. 13, 591-598 (2017).

25 Terada, Y., Yoshida, S., Takeuchi, O. \& Shigekawa, H. Real-space imaging of transient carrier dynamics by nanoscale pump-probe microscopy. Nat. Photonics 4, 869-874 (2010).

26 Yoshioka, K. et al. Real-space coherent manipulation of electrons in a single tunnel junction by single-cycle terahertz electric fields. Nat. Photonics 10, 762-765 (2016).

27 Li, S. W., Chen, S. Y., Li, J., Wu, R. Q. \& Ho, W. Joint Space-Time Coherent Vibration Driven Conformational Transitions in a Single Molecule. Phys. Rev. Lett. 119 (2017).

28 Wu, S. W. \& Ho, W. Two-photon-induced hot-electron transfer to a single molecule in a scanning tunneling microscope. Phys Rev B 82 (2010).

29 Peller, D. et al. Sub-cycle atomic-scale forces coherently control a single-molecule switch. Nature 585, 58 (2020).

30 Lobastov, V. A., Srinivasan, R. \& Zewail, A. H. Four-dimensional ultrafast electron microscopy. Proc. Natl. Acad. Sci. U.S.A. 102, 7069-7073 (2005).

31 Lee, J., Perdue, S. M., Whitmore, D. \& Apkarian, V. A. Laser-induced scanning tunneling microscopy: Linear excitation of the junction plasmon. J. Chem. Phys. 133, 104706 (2010). 
32 Hassan, M. T. et al. Optical attosecond pulses and tracking the nonlinear response of bound electrons. Nature 530, 66-70 (2016).

33 Tersoff, J. \& Hamann, D. R. Theory of the Scanning Tunneling Microscope. Phys Rev B 31, 805-813 (1985).

34 Rabi, I. I. Space quantization in a gyrating magnetic field. Phys Rev 51, 0652-0654 (1937).

35 Merino, P., Grosse, C., Roslawska, A., Kuhnke, K. \& Kern, K. Exciton dynamics of C-60based single-photon emitters explored by Hanbury Brown-Twiss scanning tunnelling microscopy. Nat Commun 6 (2015).

36 Schroder, B. et al. Controlling photocurrent channels in scanning tunneling microscopy. New J. Phys. 22 (2020).

37 Dombi, P. et al. Strong-field nano-optics. Rev. Mod. Phys. 92 (2020).

38 Born, M. \& Wolf, E. Principles of Optics, 7th Anniversary Edition. Principles of Optics, 7th Anniversary Edition, 1-952 (2019).

39 Brixner, T. et al. Two-dimensional spectroscopy of electronic couplings in photosynthesis. Nature 434, 625-628 (2005).

40 Lee, H., Cheng, Y. C. \& Fleming, G. R. Coherence dynamics in photosynthesis: Protein protection of excitonic coherence. Science 316, 1462-1465 (2007).

41 Rehm, J. M. et al. Femtosecond electron-transfer dynamics at a sensitizing dyesemiconductor (TiO2) interface. J Phys Chem-Us 100, 9577-9578 (1996). 

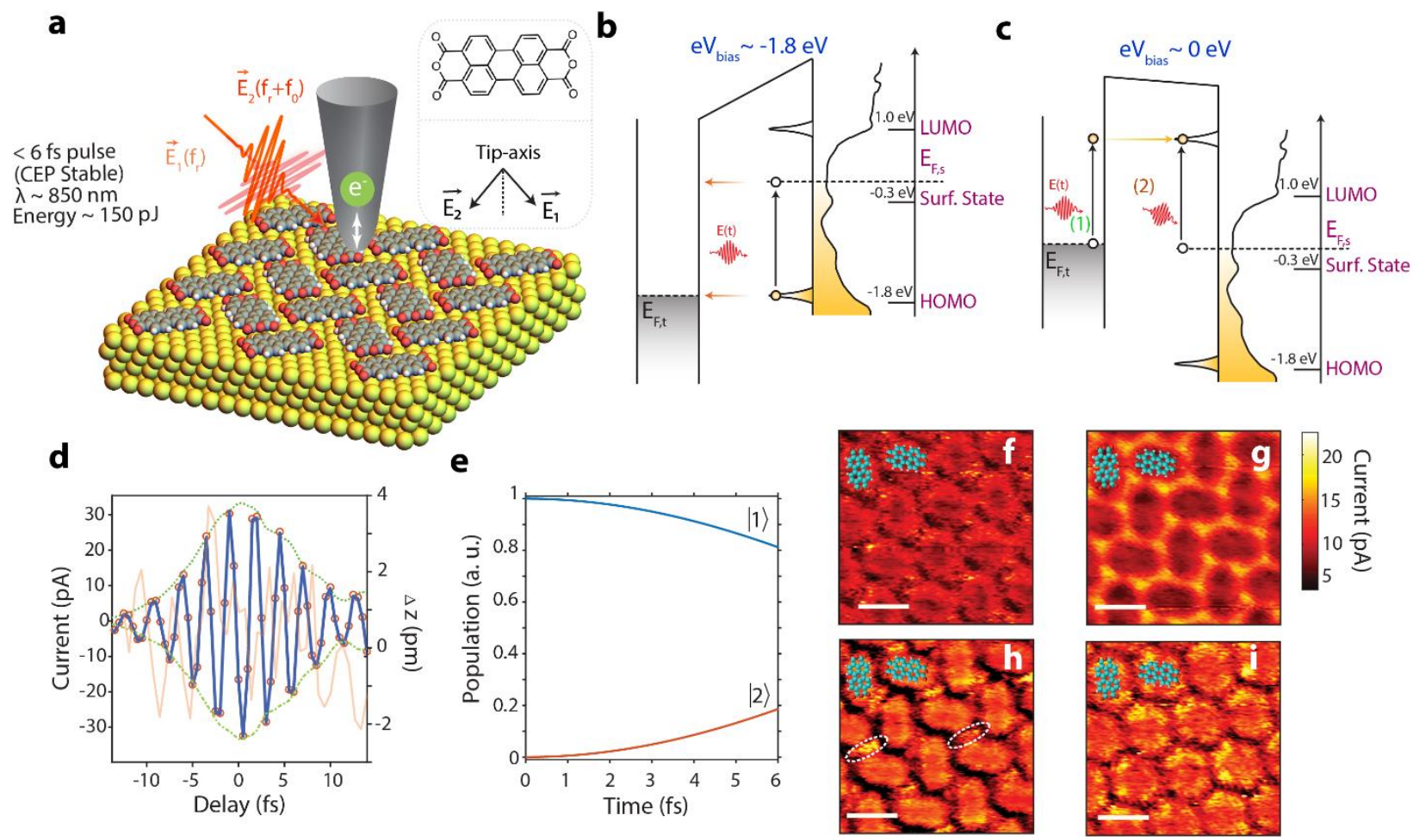

Figure 1 | Ultrashort laser-driven imaging of molecular orbitals. a, Schematic of the experimental set-up: a pair of orthogonally polarized < 6 fs carrier-envelope-phase (CEP) stable near-infrared pulses $\left(\lambda \sim 850 \mathrm{~nm}, \mathrm{I} \sim 10^{12} \mathrm{~W} / \mathrm{cm}^{2}\right)$ at slightly different carrier frequencies; $f_{r}$ (for $\mathrm{E}_{1}$ ) and $f_{r}+f_{o}$ (for $\mathrm{E}_{2}$ ) are focused at the apex of $\mathrm{W}$ nanotip in tunnel contact with a monolayer of Perylenetetracarboxylic dianhydride (PTCDA) on top of a Au (111) surface. The angle of incidence of the laser pulses is $\sim 7^{\circ}$ with respect to the $\mathrm{Au}$ (111) surface. Inset (top panel) shows the molecular structure of the PTCDA molecules. Inset (bottom panel) shows the polarization axes of the two ultrashort pulses with respect to the axis of the nanotip. $\mathbf{b}$, $\mathbf{c}$, Illustration of the laser induced transitions at the tunnel junction. At a bias where the highest occupied molecular orbital (HOMO) of PTCDA molecules is vertically lifted close to the Fermi level of the W nanotip ( $\left.E_{F, t}\right)$, photons of the laser pulse can dipole couple the HOMO of the PTCDA molecule with the surface state of $\mathrm{Au}$ (111). When the Fermi levels of the W nanotip and Au (111) surface are closely spaced, impinging photons can dipole couple either the Fermi level of the nanotip $\left(\mathrm{E}_{\mathrm{F}, \mathrm{t}}\right)$ with the lowest unoccupied molecular orbital (LUMO) of the PTCDA molecule, 'process (1)' or the surface state of the Au (111) with the LUMO of PTCDA molecule, 'process (2)'. d, Non-linear cross-correlation of the laser-induced tunneling current by orthogonally polarized laser pulses $\left(\vec{E}_{1}\left(f_{r}\right)\right.$ and $\left.\vec{E}_{2}\left(f_{r}+f_{0}\right)\right)$ at the STM junction. Here, both pulses induce a non-linear polarization along the tip axis leading to tunneling of electrons which interferes with the response from the other pulse. Variation of the tunnel gap ( $\Delta \mathrm{z}$, orange curve) during the cross-correlation measurement is shown in the right axis. e, Numerical simulation showing the temporal evolution of the population of the electronic states $(|1\rangle$ and $|2\rangle)$ separated by $\sim 1.5 \mathrm{eV}$, on exposure to a resonant 6 fs laser pulse of intensity $10^{12} \mathrm{~W} / \mathrm{cm}^{2}$, for a transition dipole moment of 0.15 a.u. along the nanotip axis direction 
(see sections I and VI in methods). f, $\mathbf{g}, \mathbf{h}, \mathbf{i}$, Spatially resolved topographic scans of single-pulse driven tunneling current in a monolayer of PTCDA molecules at various biases at the STM junction, $-1.8 \mathrm{~V}(\mathbf{f}),-20 \mathrm{mV}(\mathbf{g}),-200 \mathrm{mV}(\mathbf{h})$ and $1.4 \mathrm{~V}$ (i), respectively. Steady state STM imaging was performed in the constant current mode with a set point value of $\mathrm{I}_{\mathrm{DC}}=200 \mathrm{pA}$. White dashed ellipses in $\mathbf{h}$ are to guide the eyes of the reader to the intensity of tunneling current present in between the molecules.

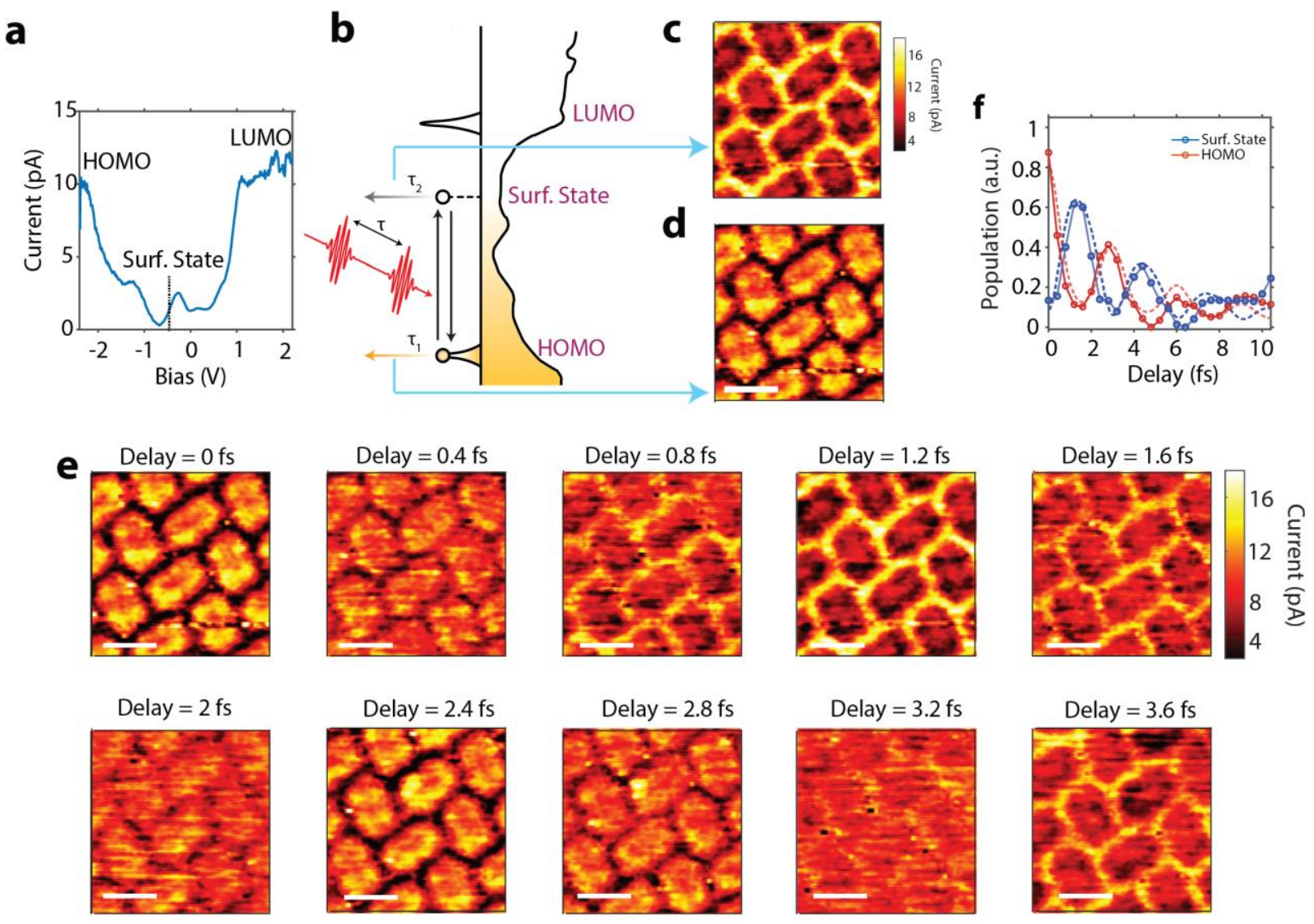

Figure 2 | Space-time resolved mapping of quantum beating. a, Differential conductance $(\mathrm{dI} / \mathrm{dV})$ measurement of a PTCDA molecule in the monolayer on the Au (111) surface. b, Schematic illustration of space-time resolved probing of quantum beating between the HOMO of the PTCDA layer and the surface state of Au (111) by delayed $(\tau)$ controlled pump and probe pulses. c, d, Spatially resolved topographic scans of the laser-induced tunneling current at fixed delays of $\tau_{2}\left(1.2 \mathrm{fs}\right.$, surface state) and $\tau_{1}(0 \mathrm{fs}, \mathrm{HOMO})$, respectively. e, Spatially resolved laserinduced tunneling current as a function of the delay between pump and probe pulses. The delay between pump and probe pulses is annotated on top of each frame. The white bar in each panel indicates a spatial dimension of $1 \mathrm{~nm}$. f, Temporal evolution of the population in the HOMO and the surface-state as a function of the delay between pump and probe pulses. Full lines: experimental results. Dotted lines: simulated temporal evolution of the population in the HOMO (red line) and the surface state (blue line) using the two-state analytical model of Eq. (1). 

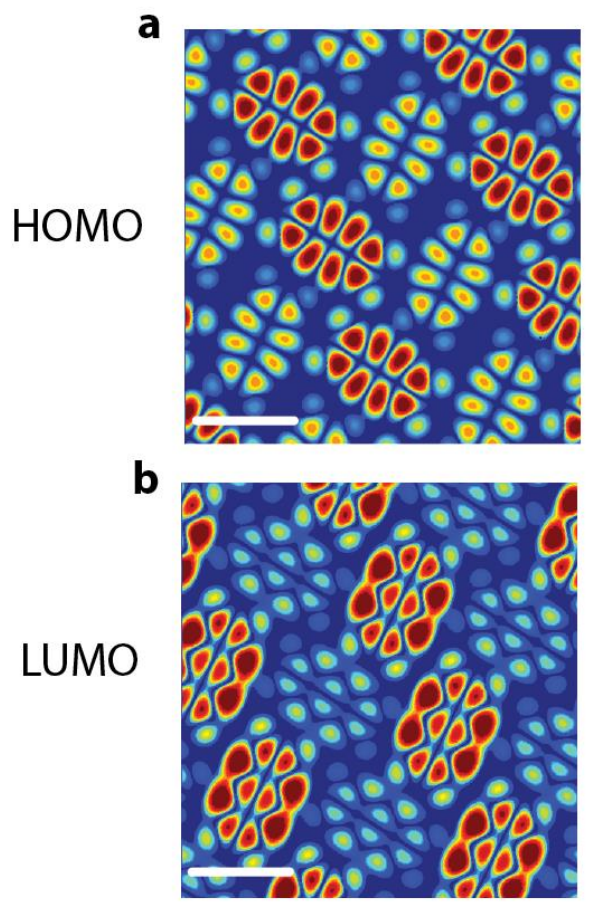
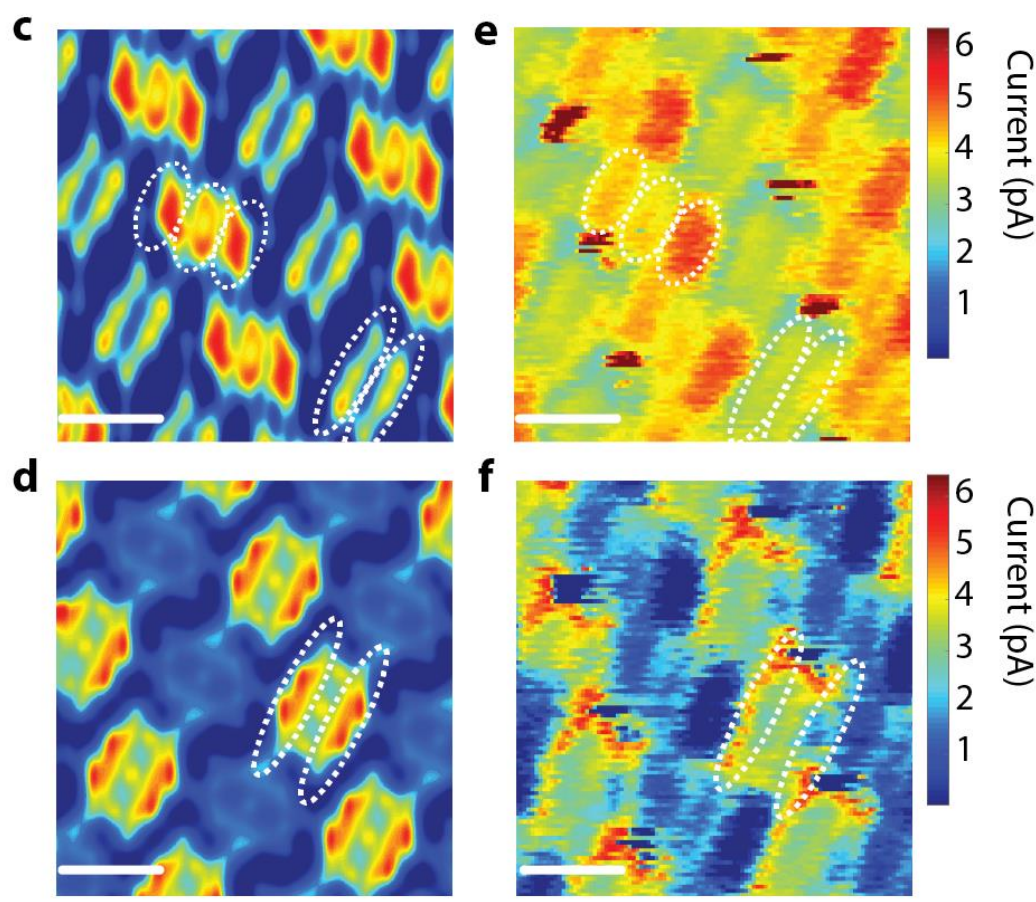

Figure 3 | Picometer resolution in laser-induced molecular orbital imaging. a, b, Simulated constant current (50 pA) STM images of the HOMO and the LUMO of PTCDA molecules constituting a 4-monolyer thick multilayer on top of Au (111), respectively, by utilizing the Tersoff-Hamann approximation (see section VI in methods). c, d, Simulated STM images obtained by convoluting the images given in $\mathbf{a}$ and $\mathbf{b}$ with an s-type wavefunction of the nanotip of the STM with a decay length of $1.8 \AA$ along the vertical direction and $0.8 \AA$ along the horizontal direction. e, f, Experimentally measured spatial distribution of the laser-induced tunneling current for the HOMO (-1.8 V) and LUMO (1.4 V) orbitals, respectively. Scale bars in all the panels denote a spatial distance of $1 \mathrm{~nm}$. White-dashed circles in $\mathbf{c}, \mathbf{d}, \mathbf{e}$ and $\mathbf{f}$ are a guide to the eyes of the reader. Steady state STM imaging was performed in the constant current mode at a set point current of $I_{D C}$ $=50 \mathrm{pA}$. 
a

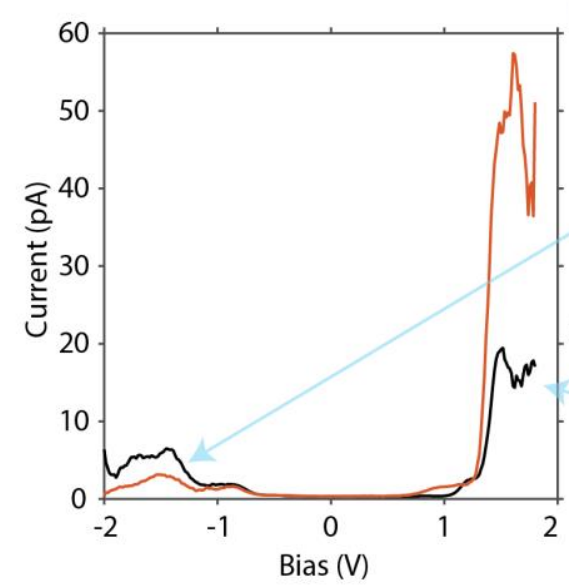

d
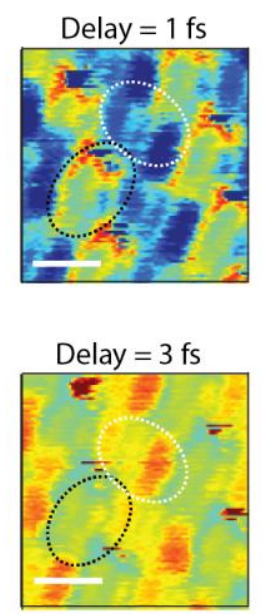

b
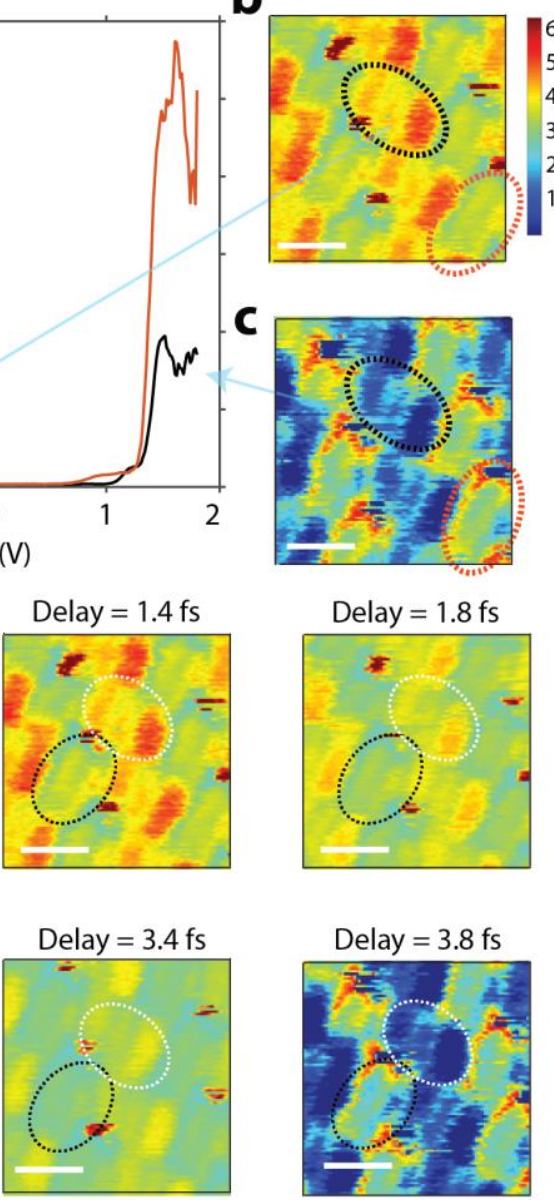
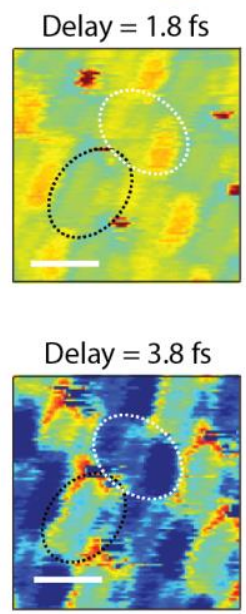
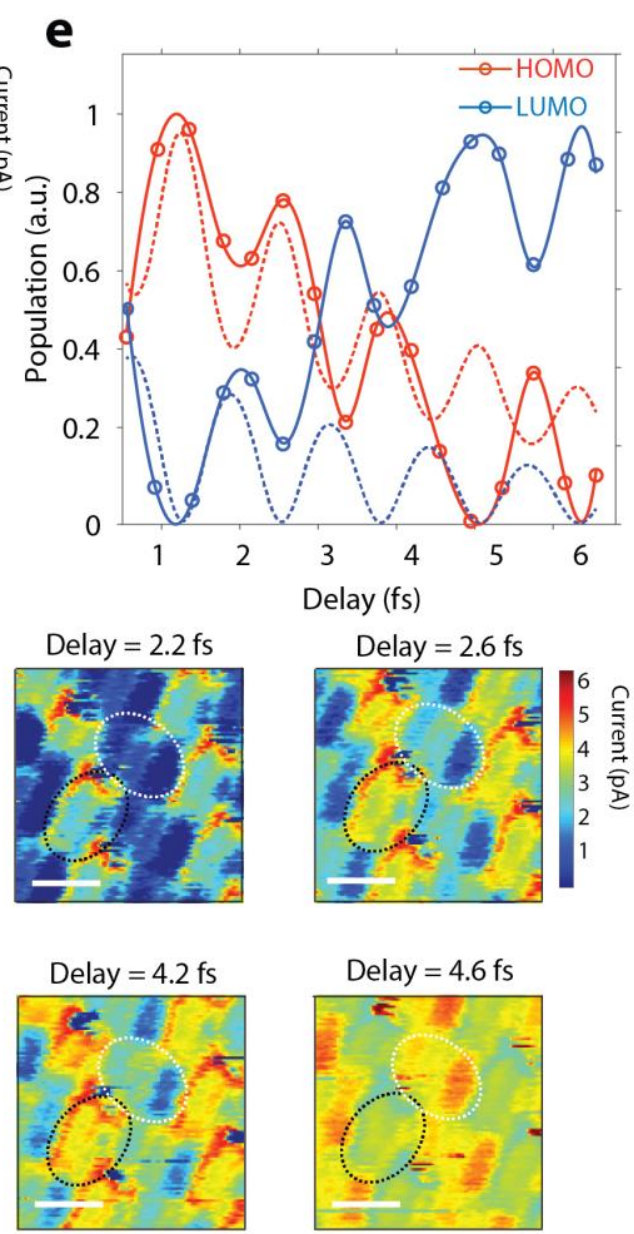

Figure 4 | Tracking valence electron motion in molecules in real-space and real-time. a, Differential conductance $(\mathrm{dI} / \mathrm{dV})$ measurement on an individual PTCDA molecule from a 4monolayer thick multilayer of PTCDA molecules on Au (111) surface. b, $\mathbf{c}$, Spatial variation of the ultrafast laser-induced tunneling current on PTCDA molecules in the 4-monoloayer coverage for the HOMO and LUMO electronic levels, respectively, at the STM junction. The density of states (DOS) for the molecules with higher and lower laser-induced tunneling current for the HOMO and the LUMO imaging is shown by the dashed black and red ellipses in $\mathbf{b}$ and $\mathbf{c}$, respectively, also indicated by light blue arrows. $\mathbf{d}$, Real-space and real-time resolved maps of the laser-induced tunneling current as a function of the delay between pump and probe pulses. The delay between pump and probe pulses is annotated on top of each frame. The white bar in each panel indicates a spatial dimension of $1 \mathrm{~nm}$. Dashed black and white ellipses in all the panels are drawn to highlight the contrast in the variation of the laser-induced tunneling current upon change of the pump-probe delay in two individual PTCDA molecules undergoing a HOMO to LUMO oscillation. e, Temporal oscillation of population in the HOMO and LUMO states as a function of delay between pump and probe pulses. Full lines: Experimental results. Dotted lines: simulated temporal evolution of the population in HOMO (red line) and LUMO (blue line) using the two state analytical model. 


\section{Figures}

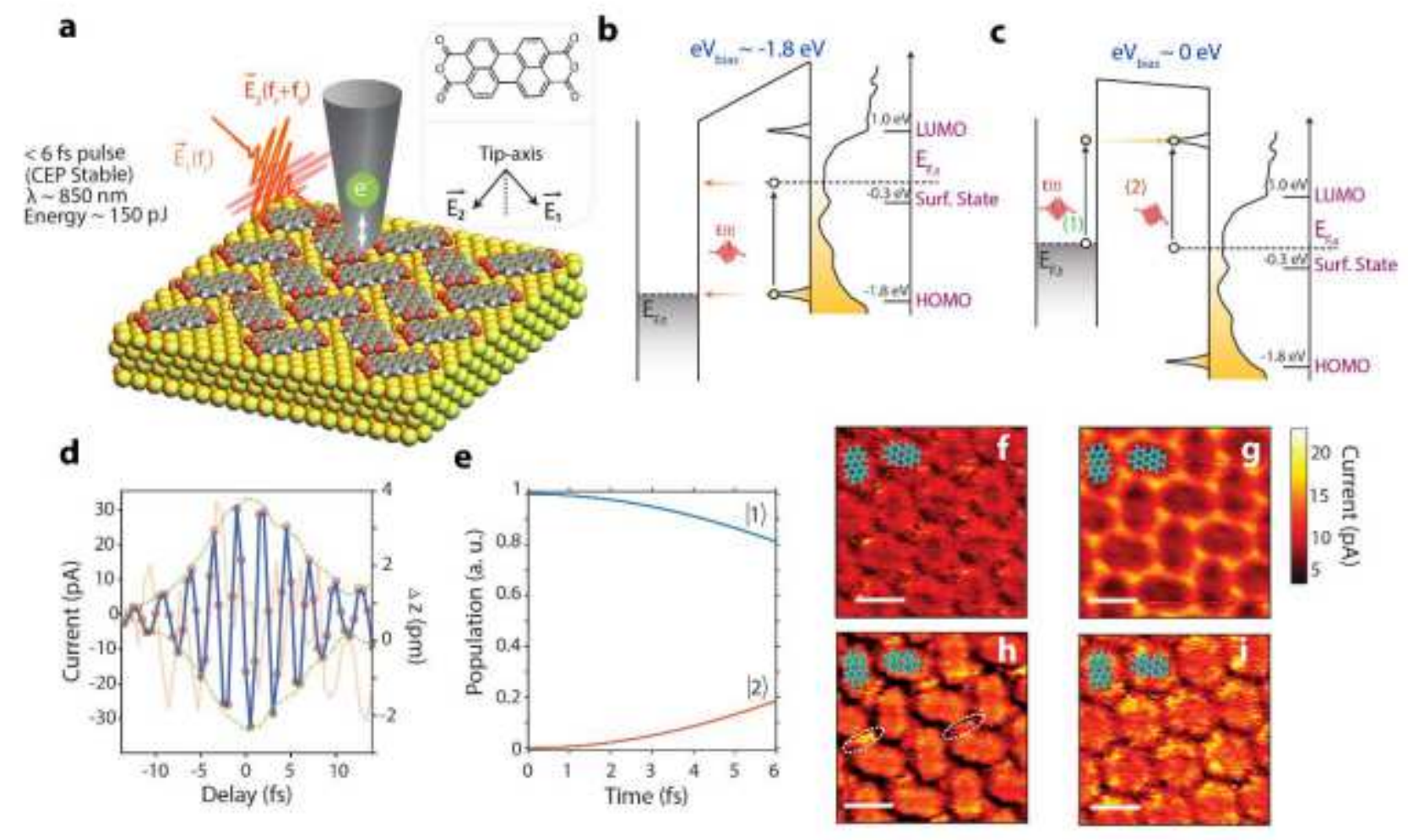

Figure 1

[Please see the manuscript file to view the figure caption.] 

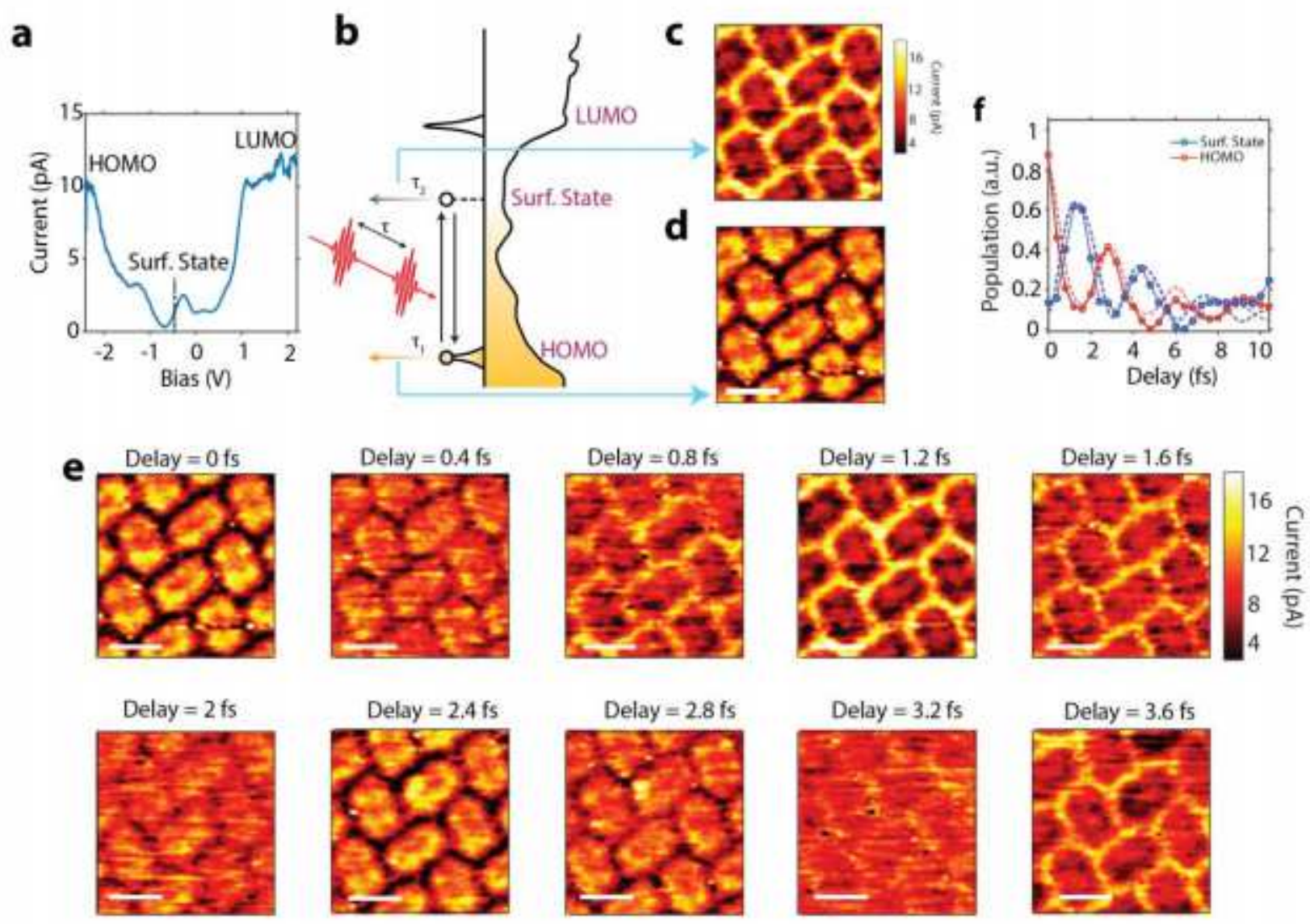

\section{Figure 2}

Space-time resolved mapping of quantum beating. a, Differential conductance (dl/dV) measurement of a PTCDA molecule in the monolayer on the Au (111) surface. b, Schematic illustration of space-time resolved probing of quantum beating between the HOMO of the PTCDA layer and the surface state of Au (111) by delayed $(\tau)$ controlled pump and probe pulses. c, d, Spatially resolved topographic scans of the laser-induced tunneling current at fixed delays of $\tau 2$ ( $1.2 \mathrm{fs}$, surface state) and $\tau 1$ ( $0 \mathrm{fs}, \mathrm{HOMO})$, respectively. e, Spatially resolved laserinduced tunneling current as a function of the delay between pump and probe pulses. The delay between pump and probe pulses is annotated on top of each frame. The white bar in each panel indicates a spatial dimension of $1 \mathrm{~nm}$. $\mathrm{f}$, Temporal evolution of the population in the $\mathrm{HOMO}$ and the surface-state as a function of the delay between pump and probe pulses. Full lines: experimental results. Dotted lines: simulated temporal evolution of the population in the HOMO (red line) and the surface state (blue line) using the two-state analytical model of Eq. (1). 

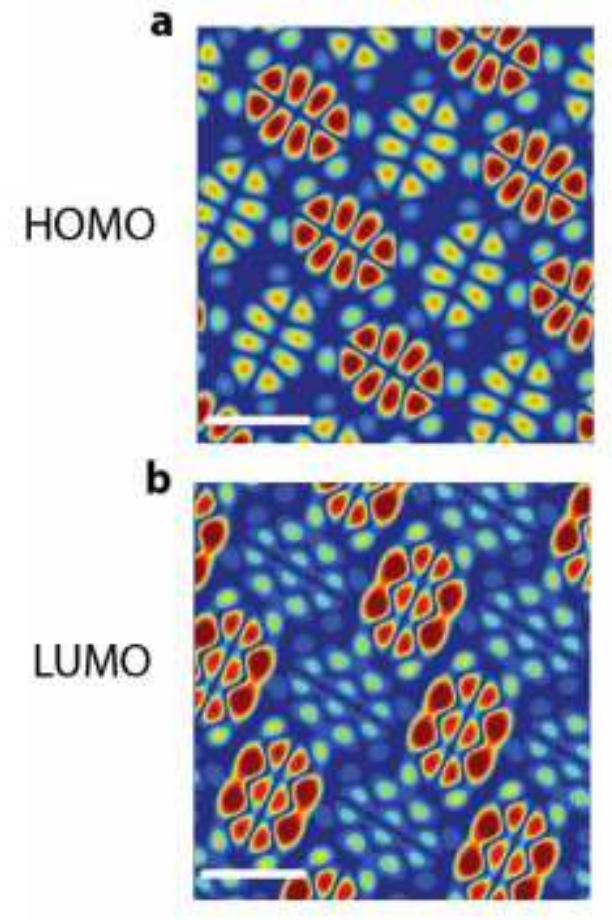

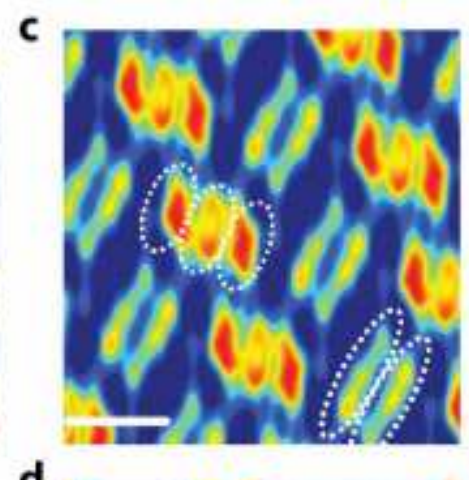

d

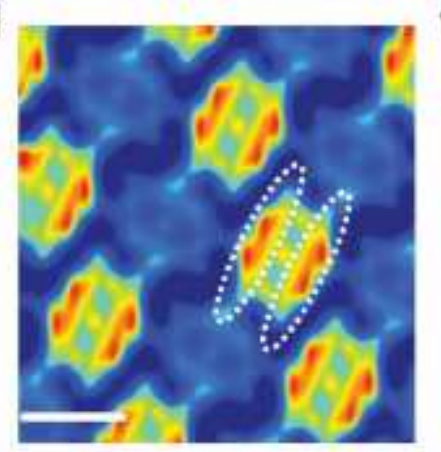

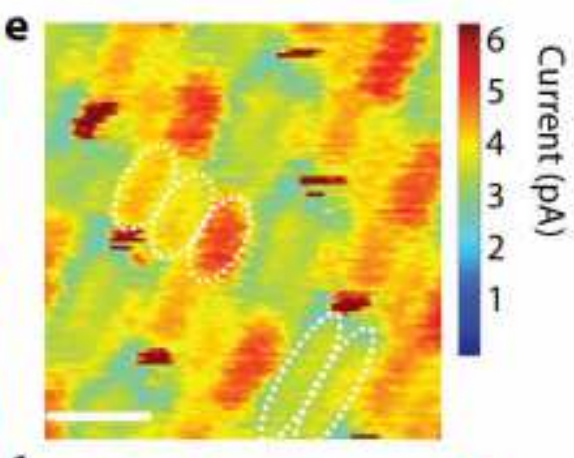

$\mathbf{f}$

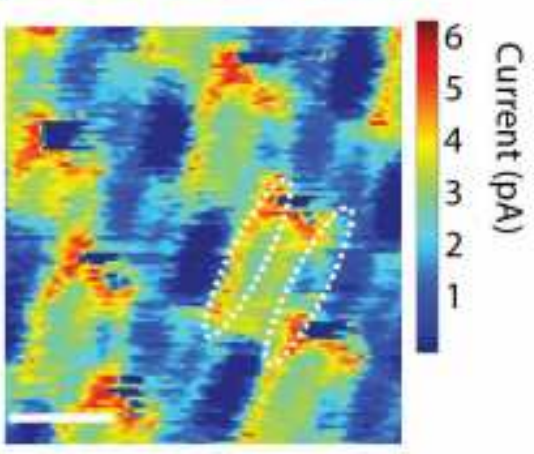

Figure 3

Picometer resolution in laser-induced molecular orbital imaging. a, b, Simulated constant current (50 pA) STM images of the HOMO and the LUMO of PTCDA molecules constituting a 4-monolyer thick multilayer on top of Au (111), respectively, by utilizing the Tersoff-Hamann approximation (see section VI in methods). c, d, Simulated STM images obtained by convoluting the images given in a and b with an stype wavefunction of the nanotip of the STM with a decay length of $1.8 \AA$ along the vertical direction and $0.8 \AA$ along the horizontal direction. e, f, Experimentally measured spatial distribution of the laser-induced tunneling current for the HOMO (-1.8 V) and LUMO (1.4 V) orbitals, respectively. Scale bars in all the panels denote a spatial distance of $1 \mathrm{~nm}$. White-dashed circles in $c, d$, e and $f$ are a guide to the eyes of the reader. Steady state STM imaging was performed in the constant current mode at a set point current of IDC $=50 \mathrm{pA}$. 
a

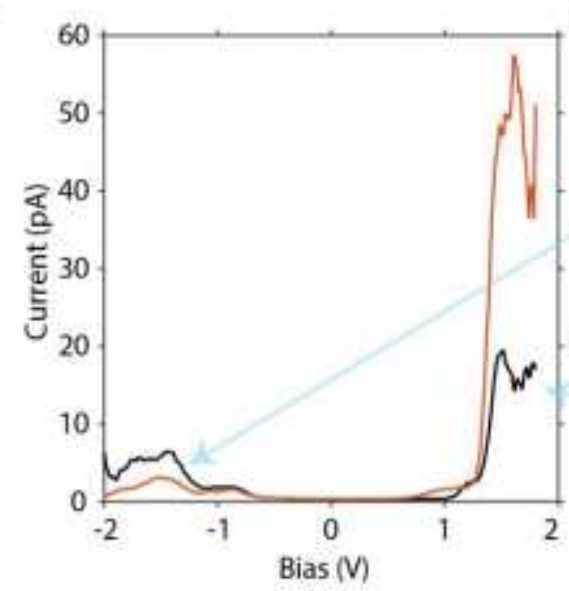

d

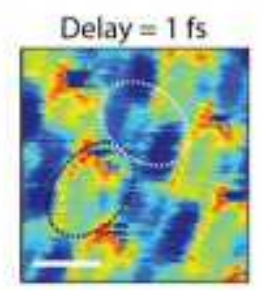

Delay $=3 \mathrm{fs}$

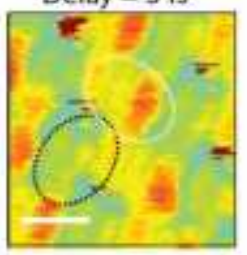

b
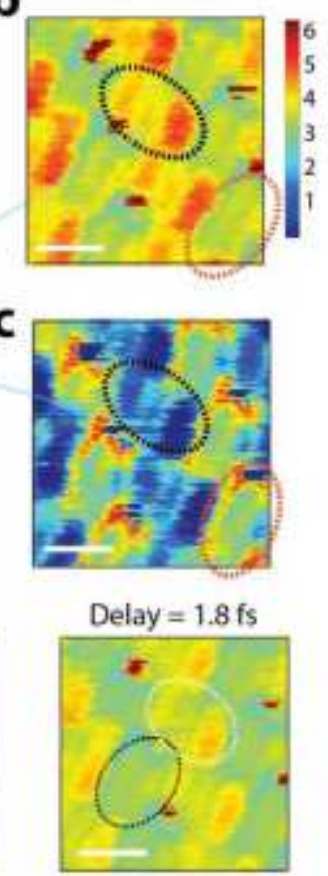

Delay $=3.8 \mathrm{fs}$

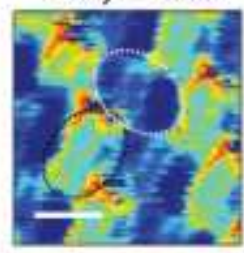

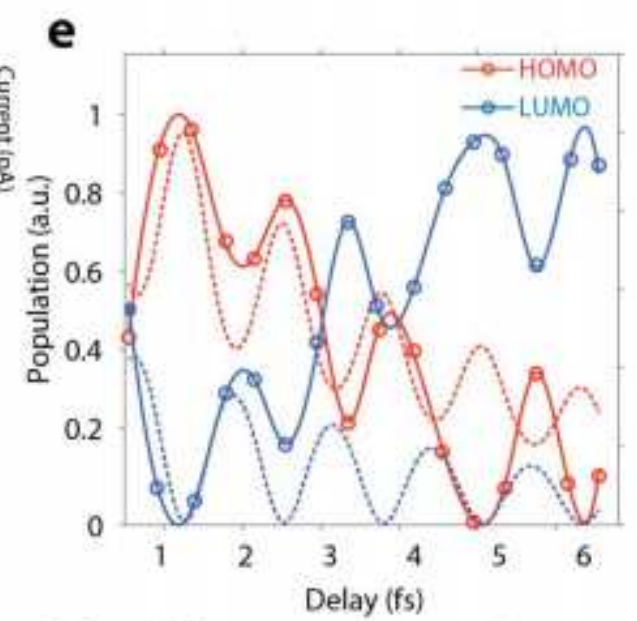
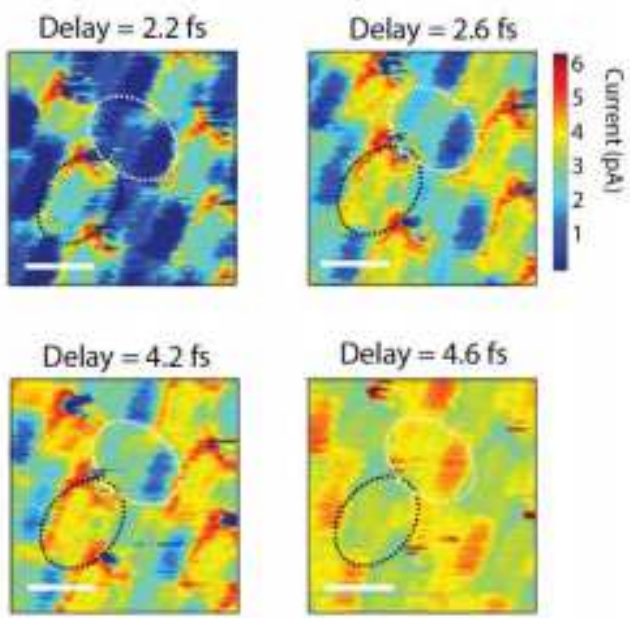

\section{Figure 4}

Tracking valence electron motion in molecules in real-space and real-time. a, Differential conductance (dl/dV) measurement on an individual PTCDA molecule from a 4- monolayer thick multilayer of PTCDA molecules on Au (111) surface. b, c, Spatial variation of the ultrafast laser-induced tunneling current on PTCDA molecules in the 4-monoloayer coverage for the HOMO and LUMO electronic levels, respectively, at the STM junction. The density of states (DOS) for the molecules with higher and lower laser-induced tunneling current for the HOMO and the LUMO imaging is shown by the dashed black and red ellipses in $b$ and c, respectively, also indicated by light blue arrows. $d$, Real-space and real-time resolved maps of the laser-induced tunneling current as a function of the delay between pump and probe pulses. The delay between pump and probe pulses is annotated on top of each frame. The white bar in each panel indicates a spatial dimension of $1 \mathrm{~nm}$. Dashed black and white ellipses in all the panels are drawn to highlight the contrast in the variation of the laser-induced tunneling current upon change of the pumpprobe delay in two individual PTCDA molecules undergoing a HOMO to LUMO oscillation. e, Temporal oscillation of population in the HOMO and LUMO states as a function of delay between pump and probe pulses. Full lines: Experimental results. Dotted lines: simulated temporal evolution of the population in HOMO (red line) and LUMO (blue line) using the two state analytical model. 


\section{Supplementary Files}

This is a list of supplementary files associated with this preprint. Click to download.

- Methods20201028.pdf 